\title{
LOGROS EDUCATIVOS DEL BACHILLER COLOMBIANO: EL CASO DE LA COHORTE DE 1978*
}

\author{
Eduardo Vélez Bustillo**
}

Pablo Luis Trouchón M. ${ }^{* * *}$

\section{Introducción}

La diversificación de los planes de estudio en la educación secundaria, con la intención de enfatizar las asignaturas vocacionales para que sean tan valoradas como las del currículo tradicional de corte académico y para que se integre la enseñanza de unas y otras, se ha constituido en política educativa en varios países del Tercer Mundo desde la década pasada. En Colombia la aparición de la educación diversificada comenzó a darse organizadamente a partir de 1970 con la puesta en marcha de los Institutos Nacionales de Educación Media Diversificada - INEM -. Desde entonces sólo se habían producido en el país esfuerzos parciales y de poca cobertura para evaluar la aplicación de la diversificación. Este documento hace parte de un esfuerzo de gran cobertura para evaluar totalmente los efectos de la educación diversificada entendida ésta como el intento de llevar al aula experiencias laborales que conjuntamente con las actividades académicas, permitan al estudiante el conocimiento del mundo del trabajo y la preparación para ingresar al mercado laboral y/o a la educación postsecundaria con unas bases educativas que no son cubiertas por el bachillerato académico clásico.

Varias son las razones que han llevado a los distintos gobiernos a estimular la diversificación en planteles que no sean orientados sólo hacia lo académico así como tampoco simples centros de entrenamiento en actividades vocacionales. Como el currículo diversificado enlaza la educación académica tradicional con la educación vocacional, le permite al estudiante acercarse a conocimientos y actitudes particulares en adición a los conocimientos propios del bachillerato académico. Es decir, el alumno en el bachillerato diversificado tiene un más amplio espectro de escogencias hacia el futuro y es por esto que su implementación ha tenido un alto nivel de popularidad en países en desarrollo

Los criterios utilizados para definir la educación media diversificada se basan en diferencias estructurales de las escuelas (académica, vocacional, comprensiva) o en las áreas curriculares ofrecidas (académica, técnica, industrial, comercial, etc.) y en el contenido del proceso de aprendizaje. Estas diferencias, que generan más expectativas y actitudes hacia el trabajo, deben inducir al egresado del bachillerato diversificado más hacia el trabajo que al bachiller académico tradicional pero sin descuidar la continuación de estudios superiores en áreas relacionadas. En efecto se supone que el bachillerato diversificado genera expectativas y logros educacionales diferenciados por área de estudio; mayor propensidad al trabajo y demanda menos educación universitaria.

\footnotetext{
* Este articulo forma parte del trabajo "Evaluación del Rendimiento Externo del Bachillerato Colombiano" adelantado y financiado conjuntamente por el MEN, el Banco Mundial y el instituto SER con la dirección de George Psacharopoulos. Con la colaboración de Olga de Amézquita, María Cecilia Suárez y Rodrigo Cuéllar.

** Sociólogo, Ph. D. Investigador Instituto SER.

${ }^{* * *}$ Funcionario del Ministerio de Educación en la División de Educación Media Diversificada. 
Ahora bien, aunque la justificación de esta variación de la educación media ha sido muy documentada; (ver Ahmed y Coombs, 1975; King, 1978; Weeks, 1978; Hurst, 1978; Haddad, 1979) es muy poca la evidencia empírica que existe con respecto a los resultados de la misma. En este artículo precisamente queremos presentar resultados de una investigación sobre la evaluación del rendimiento externo del bachillerato colombiano (Ver MEN-SER, 1982) y precisar acerca de los logros educacionales (post-secundarios) del bachiller colombiano.

\section{Antecedentes}

En el sistema educativo colombiano cualquier modalidad o tipo de bachillerato del que egresen los bachilleres permite a los mismos elegir un programa educativo de nivel intermedio o superior. El mayor problema en relación con este aspecto para los egresados que terminan la enseñanza media, es la escogencia del programa que continuaran y la búsqueda de una universidad $u$ otro instituto de educación superior que lo ofrezca. Este problema es más sentido entre los llamados bachilleres académicos, puesto que se espera que para otras modalidades el egresado escogerá más fácilmente aquellas carreras afines con su especialidad, cosa que en muchos casos no ocurre, por múltiples razones; la más destacada de ellas es que la oferta de cupos en las universidades colombianas es inferior a la demanda, lo que en muchos casos obliga a los bachilleres a ubicarse en aquella carrera o programa de más fácil ingreso pero que no está de acuerdo con sus aspiraciones e intereses. De allí provienen en gran medida los problemas de adaptación, de bajos rendimientos y pronta deserción.

Algo similar —en cuanto a oferta y demanda de empleo- sucede en el campo ocupacional, lo que en muchos casos hace que el egresado se ubique en el primer empleo que se le presente, sin tener en cuenta su preparación, experiencia, intereses y aspiraciones tanto salariales como de status dentro de la pirámide ocupacional.

Situaciones como las descritas han sido definitivas para que distintos gobiernos, a partir de 1968, hayan impulsado reformas a la educación con el fin de que los bachilleres tuvieran la doble oportunidad de continuar en el sistema educativo o pasar al sistema, ocupacional. Para ello se estableció la enseñanza media diversificada en 1969 y se ha expandido el cubrimiento de la diversificación, desde 1974, mediante el Decreto 080.

El artículo primero del Decreto 080 define la Educación Media como “... la etapa de formación educativa, posterior a la educación elemental, durante la cual el alumno tiene la oportunidad de complementar su formación integral, identificar sus intereses, aptitudes y habilidades y capacitarse prácticamente para continuar estudios superiores o desempeñar más eficientemente una determinada función en la comunidad”. En el artículo segundo uno de los objetivos allí expresados para la educación medía hace relación con la formación académica y vocacional de tipo general para que al estudiante se le habilite para la continuación de estudios superiores o para desempeñarse en una ocupación.

La necesidad de obtener información que permitiera establecer lo que ha sucedido con las reformas adelantadas, indicó la urgencia de realizar una evaluación del rendimiento externo del bachillerato colombiano, la cual contempló como objetivo fundamental la identificación de los logros educativos que por área o tipo de bachillerato han alcanzado los egresados en 1978*.

\footnotetext{
* Esta identificación es parte del estudio que conjuntamente realizan el Banco Mundial, el MEN y el Instituto SER y que incluye un seguimiento longitudinal social a una cohorte de bachilleres de 1981.
} 


\section{Metodología}

Los resultados aquí presentados se obtienen de un seguimiento realizado en 1981 entre 1.826 estudiantes graduados en 1978. Como se trata de comparar logros de estudiantes por área de estudio se seleccionó una muestra que tiende a igualar las posibilidades de selección para egresados de seis áreas diferentes.

La distribución de la muestra de bachilleres a quienes se les aplicó la encuesta por áreas es la siguiente:

\begin{tabular}{|l|r|r|}
\hline \multicolumn{1}{|c|}{ Área } & $\%$ & $\mathrm{~N}$ \\
\hline Académico & 25.1 & 458 \\
\hline Comercial & 20.0 & 365 \\
\hline Promoción social & 8.7 & 158 \\
\hline Pedagógico & 137 & 250 \\
\hline Industrial & 20.2 & 369 \\
\hline Agropecuaria & 12.4 & 226 \\
\hline
\end{tabular}

De los 1.826 egresados 1.521 (83.3\%) cursaron la primaria en una institución pública y $299(16.4 \%)$ la hicieron en una institución privada; el $52.1 \%$ de los entrevistados son hombres y la edad promedio de la muestra es de 21.8 años. Sólo el $10.2 \%$ de los padres había finalizado el bachillerato y el ingrese familiar promedio era de $\$ 28.439$ mensuales, en el momento de la entrevista.

La muestra tiene una amplia cobertura geográfica ya que se seleccionaron 25 ciudades, necesarias para completar las cuotas de las áreas de Agropecuaria y Promoción Social. En cada una de estas ciudades se escogieron, además de los INEM —cuando habíacolegios oficiales que ofrecieran las distintas áreas o tipos de bachillerato y algunos colegios privados con áreas distintas a la Académica*. La muestra en cada ciudad se conformó estratificando por área con fracciones de muestreo proporcionales al tamaño de cada área en 1980.

\section{Análisis}

\section{Actividades después del Bachillerato}

Una vez finalizado el bachillerato, el $52.5 \%$ de los egresados se dedicó al estudio aunque el $26.2 \%$ lo hacía de manera compartida con el trabajo. De todas formas es una proporción considerable ya que la muestra tiene un sesgo muy grande hacia las áreas de bachillerato diversificado. Cuando se controla por ellas (ver Cuadro 1), se notan diferencias sustanciales.

Como es de esperar los egresados del área académica se destacan por ser quienes más se dedicaron al estudio. En el otro extremo de la polarización estudio-trabajo, aparecen los egresados del bachillerato agropecuario donde cerca de la mitad, el $49.3 \%$, se dedicó exclusivamente al trabajo. Los egresados del área pedagógica (43.6\%) y del área industrial $(43.4 \%)$ también se destacaron por su orientación hacia trabajo aunque porcentajes considerables siguieron sus estudios post-bachillerato. En general, se puede

\footnotetext{
* Es necesario destacar que los INEM ejemplifican un modelo de educación diversificada que amerita ser comparado con los de otros planteles.
} 
concluir a partir de la información del cuadro No. 1 que el bachillerato diversificado genera expectativas hacia el estudio y hacia el trabajo de manera diferente al bachillerato clásico.

Un aspecto que interesa al estudio es la comparación de los bachilleres INEM por área con sus similares de otros planteles. Con respecto al punto materia de este aparte es conveniente señalar que no se observa una influencia institucional relevante sobre la dedicación post-secundaria de los egresados. Sin embargo, sí se detectan en la muestra, algunas tendencias que a continuación se presentan por área:

Área Académica: Los egresados de esta área del INEM tienden a estudiar un poco más que los académicos de otros planteles ya que $47.5 \%$ de aquellos se dedica al estudio y sólo lo hace $36.8 \%$ de éstos. La diferencia se explica porque cerca del $5 \%$ más de egresados NO INEM se dedica sólo a trabajar o a otras actividades.

Área Comercial: En esta área se observa que los egresados de planteles NO INEM tienden a estudiar en mayor proporción. Los egresados INEM de esta área parecen estar más orientados hacia el trabajo ya que el 38.0 por ciento de éstos se dedica exclusivamente al trabajo contra $33.1 \%$ de los de otros planteles.

Área Industrial: Se observa una diferencia notoria en el mismo sentido de la anterior. La orientación al trabajo de los egresados INEM es mayor ya que el $47.4 \%$ de éstos se dedica al trabajo contra el $37.8 \%$ de los otros planteles. Ambos grupos tienen un porcentaje relevante en la categoría de quienes trabajan y estudian al mismo tiempo, (26.8\% de INEM contra $29.5 \%$ de NO INEM). Por último se observan porcentajes altos dedicados con exclusividad, al estudio ya que uno de cada cuatro egresados NO INEM y un poco más de uno entre cada cinco egresados INEM lo hacen.

CUADRO No. 1

ACTIVIDAD POST-BACHILLERATO, POR AREA DE ESTUDIOS

\begin{tabular}{|l|c|c|c|c|c|c|}
\hline Área & Estudiar & $\begin{array}{c}\text { Estudiar } \\
\mathbf{y} \\
\text { trabajar }\end{array}$ & Trabajar & Otra & & \\
\hline Académico & 188 & 109 & 106 & 55 & & \\
\hline Comercial & $(41.0)$ & $(23.8)$ & $(23.1)$ & $(12.0)$ & $=$ & $100 \%$ \\
\hline & 79 & 126 & 130 & 30 & & \\
\hline $\begin{array}{l}\text { Promoción } \\
\text { social }\end{array}$ & 42 & $(34.5)$ & $(35.6)$ & $(8.2)$ & & \\
\hline & $(26.6)$ & $(22.2)$ & $(31.0)$ & $(20.3)$ & & \\
\hline Pedagógica & 37 & 87 & 109 & 17 & & \\
\hline & $(14.8)$ & $(34.8)$ & $(43.6)$ & $(6.8)$ & & \\
\hline Industrial & 84 & 103 & 160 & 22 & & \\
\hline & $(22.8)$ & $(27.9)$ & $(43.4)$ & $(6.0)$ & & \\
\hline Agropecuario & 50 & 18 & 111 & 46 & & \\
\hline & $(22.2)$ & $(8.0)$ & $(49.3)$ & $(20.4)$ & $=$ & $100 \%$ \\
\hline
\end{tabular}

Área Agropecuaria: Aquí por segunda vez se observa que los egresados INEM tienden más al estudio, mientras que los egresados de otros planteles se orientan más hacia el trabajo. Un porcentaje alto de estos últimos se dedica a actividades no 
relacionadas ni con el estudio ni con el trabajo. En efecto se encontró que el $56.4 \%$ de los egresados de planteles NO INEM se dedicó al trabajo contra sólo el $35.5 \%$ de los egresados INEM, la tendencia de estos últimos es mayor hacia el estudio, y se observó que el $36.8 \%$ de ellos se dedica exclusivamente al estudio y que el $13.2 \%$ lo comparte con el trabajo contra el $14.8 \%$ y el $5.4 \%$ respectivamente de los egresados NO INEM. Conviene destacar que los egresados del bachillerato agropecuario NO INEM provienen de zonas más rurales que aquellas de donde provienen los de los INEM. Es en las zonas más rurales donde se generan las fuentes de trabajo para los agropecuarios. Paradójicamente se observa que aunque son los que más trabajan, los egresados NO INEM presentan el mayor porcentaje de dedicación a las actividades del hogar o a no productivas; el $23.5 \%$, se dedicó a actividades diferentes al estudio y el trabajo contra sólo el $14.5 \%$ de los egresados de agropecuaria en los INEM.

Área Promoción Social: Los egresados INEM son los que presentan en esta área una mayor tendencia hacia el estudio. El $29.2 \%$ se dedicó a estudiar inmediatamente finalizó el bachillerato, mientras que sólo el $18.4 \%$ de los egresados NO INEM lo hizo. Se observa también que es mayor el porcentaje de los egresados INEM que combinan el estudio con el trabajo y que los egresados de Promoción Social de planteles NO INEM tienden a dedicarse con exclusividad al trabajo en porcentajes sensiblemente superiores a los egresados INEM.

\section{Estudio post-bachillerato}

En este aparte se presentan, primero por área y luego por área y tipo de institución, los niveles y campos de los estudios realizados por los egresados tanto inmediatamente después del bachillerato como en el momento de la entrevista.

Inicialmente se presenta la información relacionada con los niveles de estudio inicial y los realizados al momento de la aplicación de la encuesta, diferenciados primero por área y después, por área y tipo de institución. (INEM versus NO INEM). En el Cuadro 2 se presentan los niveles alcanzados por aquellos egresados (cerca del $50 \%$ ) que, después de finalizar la secundaria, se dedicaron a estudiar en uno de los niveles allí identificados.

Las diferencias que se notan son sustantivas. Se observa por ejemplo, que el nivel preuniversitario está cubierto básicamente por el bachillerato académico, el más orientado hacia los estudios universitarios. El nivel intermedio es cubierto en su orden por egresados de industrial, académico y comercial. El universitario, por egresados académicos, comerciales, industriales y pedagógicos. Los egresados académicos también tienen una alta frecuencia en cursos de capacitación: los acompañan en este nivel, los egresados de comercial e industrial. Se observa además que los académicos y comerciales se dedican básicamente a los estudios universitarios y de alguna forma, a capacitación. Los egresados de promoción social, a la inversa, llegan en mayor número a los cursos de capacitación y en segunda instancia a la universidad. Por último los pedagógicos, agropecuarios e industriales que estudian, en su gran mayoría -entre el $77.9 \%$ y el $60.2 \%$ - se dedican a los estudios universitarios y sólo en segundo y tercer plano a estudios de capacitación e intermedios. 
CUADRO No. 2

NIVEL DE LOS ESTUDIOS INICIALES POR AREA DE BACHILLERATO

\begin{tabular}{|l|c|c|c|c|}
\hline Área de bachillerato & Preuniversitario & Intermedio & Universitario & Capacitación \\
\hline Académico & 7 & 25 & 169 & 87 \\
\hline & $(2.4)^{*}$ & $(8.7)$ & $(58.7)$ & $(30.2)$ \\
\hline Comercial & $(58.3)^{* *}$ & $(26.6)$ & $(31.0)$ & $(34.4)$ \\
\hline & --- & 20 & 113 & 61 \\
\hline & --- & $(10.3)$ & $(58.2)$ & $(31.4)$ \\
\hline Promoción social & -- & $(21.3)$ & $(20.7)$ & $(24.1)$ \\
\hline & 2 & 4 & 26 & 36 \\
\hline Pedagógico & $(2.9)$ & $(5.9)$ & $(38.2)$ & $(52.9)$ \\
\hline & $(16.7)$ & $(4.3)$ & $(4.8)$ & $(14.2)$ \\
\hline & 2 & 8 & 88 & 15 \\
\hline Industrial & $(2.9)$ & $(7.1)$ & $(77.9)$ & $(13.5)$ \\
\hline & $(16.7)$ & $(8.5)$ & $(16.1)$ & $(5.9)$ \\
\hline Agropecuario & 1 & 28 & 109 & 43 \\
\hline & $(0.6)$ & $(15.5)$ & $(60.2)$ & $(23.8)$ \\
\hline & $(8.3)$ & $(29.8)$ & $(20.0)$ & $(17.0)$ \\
\hline & ---- & 9 & 40 & 11 \\
\hline
\end{tabular}

* Porcentaje de Fila.

**Porcentaje de columna.

Estas tendencias se mantienen para los niveles de estudio alcanzados tres años después, con la excepción del nivel pre-universitario que desaparece. En efecto, los programas intermedios normalmente tienen una duración que oscila entre tres y cuatro años y los universitarios de cuatro a más años por lo tanto algunos egresados de esta cohorte todavía pueden estar estudiando. Por otro lado, el preuniversitario que es una etapa de preparación necesaria para aquellos que no estaban académicamente bien preparados y no pasaron directamente a un programa de educación superior, normalmente se realiza una o dos veces pero tres años después de graduado el alumno o entró a la universidad o ya desistió de seguir intentando entrar a través de la mejora del nivel académico.

A continuación se presentan las diferencias halladas por área y por institución.

Área Académica: Se observaron diferencias claras entre egresados INEM y NO INEM. Los primeros tienden a entrar directamente a la universidad $(71.1 \%$ contra $49.7 \%)$ y tres años después los porcentajes se concentran en el nivel universitario $(85.2 \%$ de los egresados INEM contra $68.5 \%$ de egresados NO INEM).

Área Comercial: Al finalizar el bachillerato hubo pequeñas diferencias porque mientras los egresados INEM tendían a dividirse entre estudiantes universitarios $(47.7 \%)$ y de cursos de capacitación (39.6\%), los egresados NO INEM se concentraban en el nivel universitario $(68.0 \%$ y sólo uno de cada cinco recibía cursos de capacitación. Ahora, en el momento de la encuesta la gran mayoría de los egresados $(70.4 \%$ de los NO INEM y $65.4 \%$ de los INEM) se dedicaba a estudios universitarios y sólo cerca del $10 \%$ de cada grupo atendía cursos de capacitación. Conviene anotar que durante los tres años 
anteriores, los bachilleres de esta área incrementaron su participación en el nivel intermedio. Parece ser que (también sucedió con los bachilleres académicos), muchos de los que al finalizar la secundaria asistieron a cursos de capacitación, han ingresado ahora, tres años después a los niveles intermedio y universitario.

Área Industrial: La situación de ésta es totalmente diferente a la de las áreas anteriores. Los egresados industriales INEM no se diferencian de los NO INEM en cuanto al nivel de estudios realizados al finalizar el bachillerato o al momento de la encuesta. En uno y otro caso cerca del $60 \%$ de los egresados que inician estudios inmediatamente después de finalizar el bachillerato lo hace en el nivel universitario, en el momento de la entrevista lo hace cerca de un $66 \%$.

Área Agropecuaria: Entre quienes estudian tanto al finalizar el bachillerato como en el momento de hacer la encuesta, la mayoría lo hace en la universidad. (Los egresados INEM tienden a ingresar en mayores proporciones). En el nivel de capacitación se observa la tendencia contraria, los egresados NO INEM tienden, en ambos momentos, a complementarse más que los de INEM.

Área Promoción Social: Inicialmente, la mayoría, pero un poco más de los egresados NO INEM se ubicó en el nivel de capacitación. El $51.8 \%$ de los egresados INEM y el $58.3 \%$ de los NO INEM estudiaban algún curso de capacitación. Alrededor de la tercera parte de los NO INEM y el 39.3 por ciento de los bachilleres INEM de esta área, entre los que estudiaban, lo hacían en el nivel universitario.

\section{Campos de estudio}

Se presenta a continuación una descripción relacionada con los campos de estudio a los que se dedicaron los egresados; primero se presenta la información acerca de los cursos de capacitación y luego se profundiza con las carreras estudiadas.

Inicialmente se presenta la información muestral relacionada con cursos de capacitación por área de estudio. Los cursos se agruparon en catorce temas que abarcan la totalidad de los cursos asistidos. Según la información presentada en el Cuadro 3 parece existir una necesidad de complementar ternas muy estrechamente relacionados con el área de los egresados. Los bachilleres agropecuarios se capacitan mayoritariamente en cursos relacionados con esa actividad. Los bachilleres industriales se capacitan de la misma manera, en temas relacionados con esa área ya que $11.7 \%$ de los cursos se refiere a máquinas y el $23.4 \%$ a la electricidad. Entre los bachilleres pedagógicos el $29.3 \%$ se capacita en educación. Entre los egresados de Promoción Social, la mayoría se capacita en temas relacionados con secretariado aunque un porcentaje considerable, $17.5 \%$, lo hace en el área de la salud y nutrición. Los egresados de comercial y del bachillerato académico en su mayoría, $53.2 \%$, se capacitan en temas relacionados con secretariado.

Al diferenciar los egresados INEM y NO INEM, se encontraron los patrones que a continuación se presentan por área de estudio.

Área Académica: No se encontraron diferencias significativas entre los egresados INEM y los de otros planteles de esta área. La mayoría de ambos, toma cursos relacionados con secretariado y afines. 
Área Comercial: Como en el caso anterior, en ésta no se encontraron diferencias entre los egresados INEM y los egresados de otros planteles. La mayoría de ambos, asiste a cursos de secretariado, mecanografía y afines.

Área Industrial: Aunque no hay diferencias notorias vale la pena mencionar que los egresados industriales INEM tienden a tomar, en mayor intensidad que los NO INEM, cursos en el área de electrónica, radio, T.V. y afines.

Área Agropecuaria: En contraste con las anteriores áreas en ésta se encontraron algunas diferencias. Se destacan las relacionadas con técnicas agropecuarias, educación y secretariado y afines. Con respecto a la primera, mientras que $32.4 \%$ de los egresados NO INEM ha asistido a estos cursos, sólo lo ha hecho un 19.2\% de los egresados INEM. Con respecto a educación, mientras que un $16.9 \%$ de los egresados NO INEM manifestó haber tomado cursos en el área de educación, sólo un $7.7 \%$ de los egresados INEM lo ha hecho. Con respecto a secretariado mientras que sólo un 15.5\% de egresados NO INEM ha tomado cursos en secretariado y comercio, un $34.6 \%$ de egresados INEM manifestó haberlo hecho. Esta información confirma el comentario que se presentaba inicialmente sobre la diferencia urbano-rural de los egresados NO INEM e INEM de esta área. Los últimos -más urbanos - tienden a complementar sus estudios en el área comercial para poder competir en el mercado laboral de las ciudades. Los NO INEM -más rurales- se refuerzan en técnicas agropecuarias o profundizan en el área de educación ya que una de sus posibles fuentes de trabajo es la docencia. 
CUADRO No.3

CURSO DE CAPACITACION MÁS IMPORTANTE POR AREA

\begin{tabular}{|c|c|c|c|c|c|c|c|c|c|c|c|c|c|c|}
\hline Área & Agropecuario & Arte & Educación & Salud & Sociales & Recreación & Secretariado & Administración & Turismo & Idiomas & $\begin{array}{c}1 \\
\text { Máquinas }\end{array}$ & $\begin{array}{c}2 \\
\text { Electrónica }\end{array}$ & $\begin{array}{c}3 \\
\text { Matemáticas }\end{array}$ & $\begin{array}{c}4 \\
\text { varios }\end{array}$ \\
\hline \multirow[t]{2}{*}{ académico } & 1 & 2 & 7 & 3 & 4 & & 99 & 19 & 8 & 18 & 3 & 4 & 9 & 19 \\
\hline & $(0.5)$ & $(1.0)$ & (3.6) & $(1.5)$ & $(2.0)$ & --- & $(50.5)$ & $(9.7)$ & $(4.1)$ & $(9.2)$ & $(1.5)$ & $(2.0)$ & (4.6) & $(9.7)$ \\
\hline \multirow[t]{2}{*}{ comercial } & 1 & 3 & 3 & 9 & 8 & 1 & 83 & 21 & 1 & 10 & 2 & 2 & 3 & 9 \\
\hline & $(0.6)$ & $\begin{array}{l}(1.9) \\
\end{array}$ & $(1.9)$ & (5.8) & $(5.1)$ & $(0.6)$ & $(53.2)$ & $(13.5)$ & $(0.6)$ & (6.4) & (1.3) & $(1.3)$ & (1.9) & $(5.8)$ \\
\hline \multirow{2}{*}{$\begin{array}{c}\text { Promoción } \\
\text { social }\end{array}$} & 3 & 2 & 8 & 18 & 4 & 2 & 45 & 11 & 1 & 1 & & & & 8 \\
\hline & $(2.9)$ & (1.9) & $(7.8)$ & $(17.5)$ & (3.9) & (1.9) & $(43.7)$ & $(10.7)$ & $(1.0)$ & $(1.0)$ & --- & --- & --- & $(7.8)$ \\
\hline \multirow[t]{2}{*}{ Pedagógico } & --- & 5 & 29 & 3 & 12 & 3 & 19 & 8 & 1 & 6 & --- & --- & 1 & 12 \\
\hline & & (5.1) & (29.3) & $(3.0)$ & $(12.1)$ & $(3.0)$ & (19.2) & $(8.1)$ & $(1.0)$ & $(6.1)$ & --- & --- & $(1.0)$ & $(12.1)$ \\
\hline \multirow[t]{2}{*}{ Industrial } & 1 & 3 & 4 & 1 & 5 & 1 & 20 & 10 & -- & 15 & 16 & 32 & 5 & 24 \\
\hline & $(0.7)$ & $(2.2)$ & $(2.9)$ & $(0.7)$ & $(3.6)$ & $(0.7)$ & $(14.6)$ & $(7.3)$ & --- & $(10.9)$ & $(11.7)$ & $(23.4)$ & (3.6) & $(17.5)$ \\
\hline \multirow[t]{2}{*}{ Agropecuario } & 28 & 1 & 14 & 2 & 1 & 1 & 20 & 14 & 1 & 3 & 4 & 1 & 1 & 6 \\
\hline & (28.9) & $(1.0)$ & (14.4) & $(2.1)$ & $(1.0)$ & $(1.0)$ & (20.6) & (14.4) & $(1.0)$ & (3.1) & $(4.1)$ & $(1.0)$ & $(1.0)$ & $(6.2)$ \\
\hline
\end{tabular}

Entre paréntesis aparecen los porcentajes por área.

1 Aquí se incluyen máquinas Diesel, herramientas, auto mecánica, soldadores.

2 Aquí se incluyen radio, T.V., electromecánica, redes, refrigeración, electrónica.

3 Aquí se incluyen también programación y sistemas.

4 Esta categoría incluye cursos de belleza, aviación, modistería, lectura, culinaria, etc. 
Área Promoción Social: Siguiendo el patrón o tendencia general, en esta área también se observa, pero sobre todo entre los egresados INEM, capacitación extra en mecanografía, secretariado y áreas afines. Los egresados de ambas modalidades igualmente se complementan en áreas de nutrición y salud. El $12.0 \%$ de los egresados NO INEM, tomaron su curso más importante en temas relacionados con técnicas agropecuarias, mientras que ningún egresado INEM manifestó haber recibido cursos del área agropecuaria.

Una vez descrita la estructura de los cursos de capacitación, es necesario concentrarse en los campos de estudios superiores. Se supone que la experiencia en un área de estudio durante el bachillerato debe generar en el alumno una tendencia consecuente, de forma tal que los intereses en relación con los estudios sean diferenciados y acordes con la socialización y contenido educativo recibidos. De esta manera sí se encuentra una mayoría de egresados industriales estudiando derecho o medicina se concluiría que la tendencia que se debió generar fracasó ya que lo pertinente para esta área son las ingenierías y carreras técnicas afines. Si se encuentra que la mayoría de egresados académicos estudia ingenierías, tendría que concluirse análogamente.

En este aparte es necesario analizar la información y presentar los resultados considerando el área inicialmente y luego el área y el tipo de institución. A continuación se presentan los comentarios y los resultados por área.

En relación con los egresados del área Académica conviene anotar que, aunque ellos tendrían mayor flexibilidad para hallar un programa de estudios superiores que se adecúe a su formación, es de esperar que su participación sea elevada en carreras tradicionales como Medicina, Derecho y Economía.

Los resultados encontrados en la muestra (ver Cuadro 4) señalan que los académicos se dedican, en su orden, a los siguientes campos de estudio:

Economía-Administración, Educación, Sociales-Humanidades, e Ingeniería. Este resultado hay que complementario comparando las áreas por carrera, allí se observó que los egresados académicos representan el $40.9 \%$ de los estudiantes de medicina y asimilados; el $40.4 \%$ de los estudiantes de derecho; el $37.2 \%$ de los estudiantes de sociales-humanidades; el 30.8 por ciento de los de arquitectura y el $23.4 \%$ de los estudiantes de ingeniería. Los académicos son entonces mayoría en Medicina, Sociales, Humanidades y Derecho tal como se esperaba. Hay sin embargo, un dato que confunde y se relaciona con el hecho de que la mayoría de los estudiantes de Agronomía y asimilados provienen del área académica cuando no era algo esperado.

En relación con el bachillerato comercial se observa claramente que los egresados siguen en la educación post-bachillerato la tendencia esperada ya que la mitad de los egresados de comercial que estudian lo hacen en Administración-Economía, y sólo en Sociales-Humanidades se encuentra otra presencia considerable en estos egresados. En comparación con otras áreas también se observa que por carreras, los egresados de comercial representan el $91.9 \%$ de los estudiantes de Administración-Economía; el 26.7\% de los estudiantes de Sociales-Humanidades, el $26.9 \%$ de los estudiantes de Derecho: y el $21.2 \%$ de los estudiantes de Medicina. No tienen una presencia notoria en las otras carreras.

Del bachillerato en Promoción Social también se puede decir que en general mantiene una tendencia acorde con las expectativas. Los egresados se dedican principalmente a estudiar Educación y Administración-Economía; otros porcentajes menores se dedican a 
la Medicina; a las carreras Sociales-Humanidades. Adicionalmente en donde presentan porcentajes considerables, en comparación con otros bachilleres, es en Medicina y en Educación con $13.8 \%$ y $10.7 \%$ de los estudiantes en esas carreras.

Los bachilleres pedagógicos se concentran básicamente en carreras de Educación; allí se ubica más del $50 \%$ de estos egresados. En Administración-Economía también aparecen algunos porcentajes de alguna consideración. Es importante destacar, como factor indicativo de buena internalización de los objetivos de su educación secundaria, que el mayor porcentaje entre los que estudian educación corresponda a egresados del bachillerato pedagógico.

Los bachilleres del área industrial tienden a estudiar ingenierías en mayor proporción que cualquier otra carrera. Economía-Administración le sigue en importancia. Aparte de esta tendencia, acorde con la continuidad que cabría esperar, conviene destacar el hecho de que al comparar los porcentajes por carreras el $53.8 \%$ de los estudiantes de Arquitectura, el $53.3 \%$ de los de Ingeniería y el $46.7 \%$ de los de Matemáticas están constituidos por egresados del área industrial.

Por último al considerar la información de los egresados del área Agropecuaria en la última columna del Cuadro 4 se observa que el mayor porcentaje de éstos se dedica a estudiar educación, seguidos de aquellos que estudian Ingeniería y Agronomía. Aunque estas dos últimas carreras representan una mayoría, el que individualmente las carreras relaciona das con educación sean las más estudiadas, señala una aparente desviación de los resultados esperados. Sin embargo, una de las posibles fuentes de trabajo de egresados de esta modalidad se encuentra en la docencia y consistente con esta realidad, el egresado de Agropecuaria profundiza en el campo de la educación, con la posible intención de mejorar su desempeño laboral. De otra parte y es conveniente mencionarlo, en Agronomía, los egresados de Agropecuaria sólo son segundos en número, después de los Académicos. 


\section{CUADRO No.4 \\ ESTUDIOS REALIZADOS EN EL MOMENTO DE LA ENTREVISTA POR AREA DE BACHILLERATO}

\begin{tabular}{|c|c|c|c|c|c|c|}
\hline & \multicolumn{6}{|c|}{ Área o modalidad } \\
\hline Carrera & Académico & Comercial & Promoción social & Pedagógico & Industrial & Agropecuario \\
\hline \multirow[t]{3}{*}{$\begin{array}{l}\text { Administración - } \\
\text { Economía }\end{array}$} & $61 *$ & 96 & 16 & 19 & 30 & 7 \\
\hline & $21.5^{* *}$ & 50.8 & 23.5 & 13.9 & 16.2 & 10.9 \\
\hline & $26.6^{* * *}$ & 41.9 & 7.0 & 8.3 & 13.1 & 3.1 \\
\hline \multirow[t]{3}{*}{ Medicina-salud } & 27 & 14 & 9 & 7 & 6 & 3 \\
\hline & 9.5 & 7.4 & 13.2 & 5.1 & 3.2 & 4.7 \\
\hline & 40.9 & 21.2 & 13.6 & 10.6 & 9.1 & 4.5 \\
\hline \multirow[t]{3}{*}{ Educación } & 54 & 13 & 21 & 71 & 21 & 17 \\
\hline & 19.0 & 6.9 & 30.9 & 51.8 & 11.4 & 26.6 \\
\hline & 27.4 & 6.6 & 10.7 & 36.0 & 10.7 & 8.6 \\
\hline \multirow[t]{3}{*}{ Artes } & 8 & 3 & --- & 2 & 12 & --- \\
\hline & 2.8 & 1.6 & --- & 1.5 & 6.5 & --- \\
\hline & 32.0 & 12.0 & --- & 8.0 & 48.0 & --- \\
\hline \multirow[t]{3}{*}{ Sociales-humanidades } & 35 & 27 & 8 & 13 & 8 & 3 \\
\hline & 12.3 & 14.3 & 11.8 & 9.5 & 4.3 & 4.7 \\
\hline & 37.2 & 28.7 & 8.5 & 13.8 & 8.5 & 3.2 \\
\hline \multirow[t]{3}{*}{ Matemáticas } & 9 & 2 & 1 & 3 & 14 & 1 \\
\hline & 3.2 & 1.1 & 1.5 & 2.2 & 7.6 & 1.6 \\
\hline & 30.0 & 6.7 & 3.3 & 10.0 & 46.7 & 3.3 \\
\hline \multirow[t]{3}{*}{ Ciencias naturales } & 1 & --- & $\begin{array}{ll}-- \\
--\end{array}$ & 1 & 2 & --- \\
\hline & 0.4 & --- & --- & 0.7 & 1.1 & --- \\
\hline & 25.0 & $\begin{array}{ll}-- \\
--\end{array}$ & 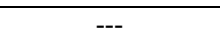 & 25.0 & 50.0 & $\begin{array}{ll}-- \\
--\end{array}$ \\
\hline \multirow[t]{3}{*}{ Derecho } & 21 & 14 & 2 & 6 & 5 & 4 \\
\hline & 7.4 & 7.4 & 2.9 & 4.4 & 2.7 & 6.3 \\
\hline & 40.4 & 26.9 & 3.8 & 11.5 & 9.6 & 7.7 \\
\hline \multirow[t]{3}{*}{ Agronomía } & 19 & 2 & --- & 4 & 4 & 11 \\
\hline & 6.7 & 1.1 & --- & 2.9 & 2.2 & 17.2 \\
\hline & 47.5 & 5.0 & --- & 10.0 & 10.0 & 27.5 \\
\hline \multirow[t]{3}{*}{ Ingeniería } & 32 & 6 & 4 & 8 & 73 & 14 \\
\hline & 11.3 & 3.2 & 5.9 & 5.8 & 39.5 & 21.9 \\
\hline & 23.4 & 4.4 & 2.9 & 5.8 & 53.3 & 10.2 \\
\hline \multirow[t]{3}{*}{ Arquitectura } & 4 & 2 & --- & --- & 7 & --- \\
\hline & 1.4 & 1.1 & $\begin{array}{ll}-- \\
--\end{array}$ & $\begin{array}{ll}-- \\
--\end{array}$ & 3.8 & $\begin{array}{ll}-- \\
--\end{array}$ \\
\hline & 30.8 & 15.4 & 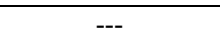 & $-\cdots$ & 53.8 & --- \\
\hline \multirow[t]{3}{*}{ Servicios } & 4 & 3 & --- & 2 & 2 & 1 \\
\hline & 1.4 & 1.6 & $\overline{---}$ & 1.5 & 1.1 & 1.6 \\
\hline & 33.3 & 25.0 & --- & 16.7 & 16.7 & 8.3 \\
\hline \multirow[t]{3}{*}{ No específicos } & 9 & 7 & 7 & 1 & 1 & 3 \\
\hline & 3.2 & 3.7 & 10.3 & 0.7 & 0.5 & 4.7 \\
\hline & 32.1 & 25.0 & 25.0 & 3.6 & 3.6 & 10.7 \\
\hline
\end{tabular}

* Número absoluto.

** Porcentaje de la columna ares.

*** Porcentaje de la fila carrera.

Pasando a la comparación por área de estudio y tipo de institución (INEM versus NO INEM) es posible interpretar la información obtenida tratando de identificar diferencias en la dirección de la educación a nivel post-bachillerato.

Área Académica: No se aprecian diferencias de consideración entre los egresados académicos del INEM y de otros planteles. Sin embargo, los egresados INEM tienden a seguir en Ingeniería y Agronomía, mientras que los NO INEM se concentran en las carreras de Medicina-Salud, Administración-Economía y Educación. En las otras carreras las diferencias registradas son mínimas.

Área Comercial: En esta área de bachillerato se aprecian algunas diferencias. Aunque en Administración-Economía, la carrera más próxima al área, no se encontraron diferencias, sí se puede ver que los egresados INEM se orientan más hacia el Derecho y la Educación Mientras que los de otros planteles se orientan hacia la Medicina. 
Área Industrial: En lo referente al área industrial, las tendencias son bastante similares y no se encuentran diferencias entre los egresados INEM y los de otros planteles. En ambos grupos hay una notoria tendencia hacia la Ingeniería y la Arquitectura.

Área Agropecuaria: Aunque hay una mayor proporción de egresados INEM estudiando Agronomía e Ingenierías, las diferencias que se aprecian no son significativas.

Área Promoción Social: Con dos excepciones, las tendencias apreciadas en cuanto a proporciones de estudio actual son muy similares para egresados de INEM y de otros planteles. Los NO INEM tienden mayoritariamente a estudiar Economía-Administración. Mientras que los egresados INEM aparecen (16.7\%) entre los dedicados al estudio de la Medicina, los de planteles NO INEM no aparecen en esta carrera. A este propósito se anota que inicialmente el $16.7 \%$ de los egresados NO INEM y el $23.2 \%$ de los de INEM estudiaban Medicina. Es evidente que ha habido una deseción significativa principalmente por parte de los alumnos NO INEM.

Para finalizar el aparte, un comentario general acerca de los cambios ocurridos por campo de estudios universitarios haciendo énfasis en la permanencia y en los cambios ocurridos. En general se observa una tendencia a permanecer en la carrera ya que los porcentajes de personas que siguen estudiando lo que inicialmente seleccionaron va desde $100.0 \%$ en Arquitectura, hasta $50 \%$ en Ciencias Naturales con un promedio de $75.96 \%$ de permanencia (Ver cuadro 5 ).

CUADRO No.5

PORCENTAJES DE ALUMNOS QUE ESTUDIAN

ACTUALMENTE LA CARRERA QUE INICIARON DESPUES DEL BACHILLERATO

\begin{tabular}{|l|c|}
\hline \multicolumn{1}{|c|}{ Carreras } & $\begin{array}{c}\text { Porcentaje } \\
\text { de } \\
\text { retención }\end{array}$ \\
\hline Administración-Economía & 77.2 \\
Medicina-Salud & 73.5 \\
Educación & 84.8 \\
Bellas Artes & 80.0 \\
Sociales-Humanidades & 53.5 \\
Matemáticas & 73.9 \\
Ciencias & 50.0 \\
Derecho & 79.5 \\
Agronomía & 75.9 \\
Ingenierías & 87.6 \\
Arquitectura & 100.0 \\
\hline
\end{tabular}

Adicionalmente es posible indicar cómo se mueven los "desertores". los de Administración-Economía tienden a cambiarse a Sociales-Humanidades y a Educación principalmente; los de Medicina-Salud se dirigen a Administración-Economía. En las otras carreras no se observan cambios sistemáticos. 


\section{Quiénes entran a estudiar: (Modelos multivariados)}

Hasta aquí se ha examinado la relación del área de bachillerato y de la institución donde se estudió con el logro educativo. Así se inició el proceso de entendimiento que permite establecer el por qué unos estudiantes alcanzan logros y otros no. Pero en adición a esas variables, existen otras que pueden considerarse para entender el proceso del logro. En efecto en la literatura especializada se han considerado, desde hace algunos años, modelos que incluyen variables indicadoras de la experiencia socioeconómica y educativa de los alumnos para tratar de explicar el nivel de logros. (Ver Jenks 1972; Psacharopoulos, Soumelis, 1979; Schiefelbein y Farrell, 1982).

Parece conveniente presentar unos ejercicios multivariados donde simultáneamente se controlan algunas variables consideradas pertinentes para entender el proceso. De esta manera se establecen los efectos independientes de cada una de las variables utilizadas y el poder de cada una de ellas por medio de la técnica de análisis discriminante (ver Van de Geer, 1971).

Este ejercicio adicionalmente producirá información que será definitiva para establecer la bondad del bachillerato diversificado y del sistema INEM en particular.

Cada situación se va a estudiar utilizando dos modelos. El primero incluye la edad, sexo, el tamaño de la familia, el ingreso de la familia, el tamaño de la ciudad, el status ocupacional del padre, si el colegio es privado o no, si es INEM o no, y el segundo incluye la última pero introduce las áreas de estudio.

La inclusión de las variables independientes tiene una justificación casi por si misma. En forma rápida sin embargo, se presentan algunas justificaciones para cada variable. La edad puede ser un factor inhibidor de la continuación de estudios superiores, entre mayor sea la persona más probable es que tenga que dedicarse a trabajar. El sexo, en una sociedad donde culturalmente el ser hombre tenga implicaciones distintas a las de ser mujer, puede condicionar los patrones de comportamiento. El tamaño de la familia igualmente puede inhibir la disponibilidad al estudio; entre más numerosa sea la familia menores serán las posibilidades de los miembros para dedicarse al estudio. El ingreso de la familia también se considera importante porque genera o da mayores posibilidades al estudiante miembro de una familia con recursos. El status ocupacional del padre* en cierto sentido, correlacionado con la anterior, se incluye porque se presume que los hijos de profesionales tenderán con mayor posibilidad a seguir estudios que hijos de artesanos o de campesinos, por ejemplo. El tamaño de la ciudad Puede tener un efecto, entre otras cosas, porque se supone que en las ciudades más grandes hay más posibilidades de estudiar que en las pequeñas. La división de los colegios en privados o públicos, genera una variable importante desde el punto de vista de la planificación administrativa, y se incluye porque se ha encontrado que los alumnos de colegios públicos tienden a estudiar menos una vez finalizado el bachillerato. La inclusión de variables como el ingreso en el mismo modelo servirá para independizar el efecto real de esta variable. Las variables que definen a los egresados INEM, y a los egresados de cada área se incluyen porque, precisamente se pretende establecer si hay logros diferenciales.

En relación con la realización de estudios inmediatamente después de finalizado el bachillerato, se presentan a continuación los dos modelos para contrastarlos. En el

\footnotetext{
* Se generó una escala partiendo de la ocupación que diferencia el status de la misma otorgándole mayor puntaje a los profesionales hasta menor status a los trabajadores rurales.
} 
Cuadro 6 se aprecia cómo el segundo modelo, que incluye las áreas, es más importante para discriminar entre quienes estudian y quienes no. El primer modelo indica que la edad es la principal variable, controlando por las demás, para determinar quién se dedica al estudio y quién no. Los menores tienden a estudiar en mayores proporciones.

Es interesante observar que los egresados INEM tienden a seguir estudiando en mayores proporciones que los de otros planteles, indicando así que a los últimos se les inculca menos la necesidad de estudio y probablemente más la del trabajo. La tercera variable que es altamente significativa en discriminar, es el status ocupacional del padre. Entre más alto sea, mayor posibilidad tiene el hijo de estudiar, señalando así el mantenimiento de un estado socio-económico pero dirigido por el status ocupacional y no por el ingreso como se verá a continuación.

Las otras variables que originalmente se incluyeron en el modelo tienen menos relievancia y su orden de importancia se observa en el Cuadro 6. Igualmente se presenta la significancia del modelo, de acuerdo con la correlación canónica y el lambda de Wilks; por último se puede ver el poder clasificatorio del modelo el cual es del $58 \%$. Ello significa que ese porcentaje de los casos es clasificado adecuadamente.

En el mismo cuadro se presenta la información con respecto al segundo modelo que parece más interesante. Se observa que al introducir las áreas de estudio, surge con bastante fuerza discriminante el bachillerato académico. Esto sirve para identificar un logro particular de esta área de estudio ya que se espera que así sea. Los bachilleres académicos independientemente de las otras características controladas, tienden a continuar sus estudios.

La edad se sigue manteniendo como inhibidor de los estudios post-bachillerato, ya que entre mayor sea el alumno, menor es la probabilidad de que siga haciéndolo. Es importante recalcar la relevancia de esta variable porque entre todas las significativas, se mantiene, aun controlando variables tan importantes como el ingreso familiar.

Otro resultado destacable es el que se relaciona con el papel de los egresados Agropecuarios y de los de Promoción Social, en ese orden. Estos alumnos tienden con gran fuerza a dedicarse al estudio lo cual se podría atribuir a necesidades de complementación o a que no consiguen trabajo por escasez de oferta.

En este modelo el tamaño de la ciudad entra a discriminar relativamente y la tendencia es que entre mayor sea la ciudad mayores son las probabilidades de estudiar para los egresados. Se anota que en el modelo anterior cuando se controlaba por INEM, la variable tamaño de la ciudad no era relevante. 


\section{CUADRO No.6 \\ MODELOS DISCRIMINANTES DE QUIENES SE DEDICAN AL ESTUDIO \\ DESPUES DE FINALIZADO EL BACHILLERATO. SOLO SE PRESENTAN \\ COEFICIENTES STANDARIZADOS SIGNIFICATIVOS AL 0.01}

\section{Variables en orden de importancia}

\section{Modelo 1}

\begin{tabular}{lr}
\multicolumn{1}{c}{ Edad } & -0.87 \\
INEM & 0.31 \\
Status ocupacional & 0.9 \\
Tamaño familia & \\
Tamaño ciudad & \\
Ingreso familia & \\
Colegio privado & \\
Sexo &
\end{tabular}

Correlación

Canónica

0.19

0.28

Lambda de Wilks

Capacidad de

Clasificación

Modelo 2

$\begin{array}{lr}\text { Bto. Académico } & 0.82 \\ \text { Edad } & 0.67 \\ \text { Bto. Agropecuario } & 0.31 \\ \text { Bto. Promoción } & 0.29 \\ \text { Tamaño ciudad } & 0.20 \\ \text { Sexo 0.16 } & \\ \text { Colegio privado } & 0.11 \\ \text { Status ocupacional } & \\ \text { Tamaño familia } & \\ \text { Bto. industrial } & \\ \text { Bto. comercial } & \\ \text { Ingreso familia } & \end{array}$

En este nuevo modelo, el sexo aparece como relevante al discriminar y el análisis induce a afirmar que los hombres, independientemente del área, la edad o cualquier otra variable, tienen mayor probabilidad de continuar estudios que las mujeres.

La última variable relevante al discriminar entre los egresados de 1978, es la referente a la naturaleza del colegio — privado o no-. En este orden de ideas, es posible afirmar que los egresados de colegios privados tienen mayor probabilidad de continuar estudios que los de colegios no privados, una vez finalizan el bachillerato. Esta variable y el sexo no aparecían como relevantes al discriminar con el primer modelo.

El segundo modelo tiene mayor capacidad discriminante, produce mayor información que el primero, tiene mayor coeficiente de correlación canónica y más alta capacidad de clasificación la cual se incrementa desde $58.50 /$ o hasta $65.9 \%$. Todo ello ayuda a entender mejor los logros diferenciados por área de bachillerato y a identificar al egresado académico como el que más logra ingresar a estudios post-bachillerato seguido a mucha distancia por el agropecuario y el de promoción social. En última instancia se encuentra a los egresados de industrial y de comercial. El pedagógico presenta poca tendencia al estudio post-bachillerato. 
A continuación se presentan los modelos que ayudarán a establecer quiénes se mantienen estudiando tres años después de haber finalizado el bachillerato. (Ver Cuadro 7).

Lo primero que se observa es que los modelos mejoran. El incremento de la correlación canónica indica que el modelo discriminante es mejor para diferenciar entre quiénes estudian tres años después de graduados y quiénes no.

Por su parte, el primer modelo sigue presentando a la edad como variable más relevante y mantiene el sentido positivo del papel de los INEM. Cambia el status ocupacional por el ingreso familiar indicando de todas maneras que cuando las familias son más acomodadas permiten mayor continuidad en los estudios post-bachillerato.

El tamaño de la ciudad entra a ser una variable significativa para determinar las posibilidades de quién puede estudiar.

El modelo dos mantiene el papel del bachillerato académico, de la edad, del tamaño de la ciudad, del sexo y del bachillerato agropecuario. El papel de este último se invierte indicando lo siguiente: inmediatamente después de finalizado el bachillerato es posible que éstos como los egresados de promoción social, se dediquen a tomar cursos de capacitación o intermedios y una vez finalizados 5at' $r$ se integran de manera exclusiva a la fuerza laboral o a actividades diferentes al estudio - Conviene resaltar el papel del sexo porque definitivamente indica la mayor participación de los hombres en los estudios postsecundarios. El bachillerato industrial y el comercial que antes mostraban tendencias no significativas hacia el estudio post-secundario, ahora aparecen como significativos con tendencia negativa lo cual se enmarca dentro de lo esperado por cuanto los egresados de esta área se dirigen hacia la fuerza laboral en mayores proporciones y permitiría señalar además que tienden a permanecer allí. 


\section{CUADRO No.7 \\ MODELOS DISCRIMINANTES DE QUIENES ESTUDIAN TRES AÑOS DESPUES DE FINALIZADO EL BACHILLERATO. SOLO SE PRESENTAN COEFICIENTES STANDARIZADOS, SIGNIFICATIVOS AL 0.01}

\begin{tabular}{|c|c|c|c|}
\hline Modelo 1 & & Modelo 2 & \\
\hline Edad & -0.85 & Edad & -0.81 \\
\hline $\begin{array}{l}\text { Tamaño de } \\
\text { la ciudad }\end{array}$ & 0.20 & Bto. Agropecuario & -0.32 \\
\hline Ingreso familiar & 0.17 & Bto. Industrial & -0.27 \\
\hline INEM & 0.13 & Sexo & 0.21 \\
\hline Privado & & Bto. Académico & 0.17 \\
\hline Status ocupacional & & Ingreso familiar & 0.14 \\
\hline Tamaño familiar & & Tamaño de la ciudad & 0.14 \\
\hline Sexo & & $\begin{array}{l}\text { Bto. Comercial } \\
\text { Privado } \\
\text { Bto. Promoción } \\
\text { Tamaño familiar } \\
\text { Status ocupacional }\end{array}$ & -0.13 \\
\hline Correlación canónica & .32 & & 0.35 \\
\hline Lambda de Wilks & .89 & & 0.87 \\
\hline $\begin{array}{l}\text { Capacidad de } \\
\text { clasificación }\end{array}$ & $63.95 \%$ & & $64.96 \%$ \\
\hline
\end{tabular}

Igualmente vale la pena destacar el papel de la edad ya que se presenta como una barrera para la continuación de los estudios. Igualmente al contrastar principalmente instituciones de educación media diversificada, los INEM parecen inducir más hacia el estudio a sus egresados en comparación con los planteles NO INEM. Comparando por área de estudio se destaca, comprobando lo esperado, el bachillerato académico como inducidor de los estudios superiores contrario al efecto del industrial y del comercial que lo restringen. Merece puntualizarse el papel desempeñado por el área agropecuaria que en un lapso de tres años produce dos efectos contrarios, al inicio del período postbachillerato parece que los egresados del área están deseosos de proseguir estudios pero al cabo de tres años sus preferencias se inclinan hacia el trabajo, producto de la realidad de sus posibilidades en la estructura educativa y ocupacional del país.

Por último se quiere mencionar que los modelos presentados son apenas algunos entre los muchos que ameritan atención y por lo tanto se debe tomar este artículo sólo como una parte de un estudio que tiene un mayor cubrimiento.

\section{Conclusión}

Sin muchas pretensiones en este artículo inicialmente planteábamos la necesidad de empíricamente constatar si el bachillerato diversificado lograba las expectativas supuestas en relación con la educación postsecundaria. Específicamente las hipótesis básicas son las que generan menos demanda por la educación postsecundaria; menos demanda por educación universitaria; pero principalmente que generan logros educacionales diferenciados por área de estudio. 
Los principales resultados observados, permiten, de una manera general, concluir acerca de las bondades de los logros educacionales del bachillerato diversificado en nuestro país. Por un lado, se puede constatar que en general, la tendencia del bachillerato académico hacia el estudio es mayor que la de las otras áreas de estudio cuyos alumnos a su vez se integran más al mercado laboral. Inclusive al utilizar modelos multivariados, que controlan por variables de la estructura socioeconómica del alumno, se observa que la diversificación tiene un impacto en la continuación de los estudios postsecundarios. Las principales características observadas revelan al bachiller clásico como un inducidor de los estudios superiores y como se ve en el Cuadro 7, los bachilleratos agropecuarios, industrial y comercial juegan el papel contrario. Esto sin embargo no quiere decir que porcentajes relevantes de estos no continúen estudios superiores, simplemente que lo hacen en menor intensidad que los académicos. Otro resultado que aiim-rita distinción es el observado con la modalidad de los INEM. Los egresados de estos institutos están más propensos a la continuación de los estudios superiores que los estudiantes de modalidades univocacionales, indicando pues que el sistema educativo genera logros diferenciales a este respecto.

Características socioeconómicas del egresado también marcan unas tendencias diferentes y entre ellas las más relevantes son las siguientes. En los Cuadros 6 y 7 se puede ver cómo el estatus social de la familia determina la continuación de los estudios. Los hijos de familias con mayores ingresos y con mayores estatus ocupacionales tienden a continuar estudios superiores en mayores proporciones que estudiantes provenientes de familias con bajos ingresos y bajo estatus ocupacional. Esto indica la manera como el estatus social se transmite de padres a hijos.

También se encuentra que los jóvenes y los hombres estudian en mayores proporciones que los mayores (posiblemente tienen más necesidades de trabajar) y que las mujeres. Esto indica que a pesar de que en los últimos años las mujeres han ingresado sustancialmente a los niveles educativos superiores, los hombres siguen haciéndolo en mayores proporciones.

Por último, aspectos como el tamaño de la ciudad y la naturaleza del colegio donde se estudia, también y de manera independiente, reflejan distintas posibilidades en relación con la continuación de los estudios. En las ciudades más grandes, por haber una infraestructura mejor y mayor número de posibilidades educativas se estudia más. $Y$ egresados de colegios privados también son más susceptibles de continuar estudios superiores.

En relación con los logros diferenciales también se encontró un resultado que permite concluir la obtención de un logro educativo del bachillerato diversificado. Simplemente el hecho de que los egresados del bachillerato industrial estudien preferencialmente ingenierías; de que los egresados de comercial estudien preferencialmente administración o economía; de que los egresados de pedagógico estudien educación; de que los egresados de promoción social estudien educación, administración o medicina; y de que los académicos no tengan preferencias notorias, sirve para indicar la obtención del logro diferencial.

Por último en cuanto al nivel de estudios, también se observa la tendencia esperada. Los académicos se orientan más hacia el nivel universitario, aunque muchos sólo toman cursos de capacitación. Los industriales aunque en su mayoría se integran al nivel universitario son quienes más presencia tienen en el nivel intermedio. Los comerciales tienen bastante tendencia a la educación intermedia y/o a la capacitación. Los de 
promoción social se concentran en el nivel de capacitación. Los agropecuarios y los pedagógicos son quienes menos parecen marcar la tendencia esperada ya que se sitúan mayoritariamente en el nivel universitario.

Para concluir, las tendencias de los indicadores utilizados permiten identificar unos logros positivos de la educación media diversificada.

Los indicadores utilizados en este artículo son sólo unos de muchos que deben ser tenidos en cuenta antes de concluir categóricamente sobre las bondades de los logros educativos de la educación media diversificada. 


\section{BIBLIOGRAFIA}

DAVIS, Russell G. Planning Education for Development: Vol. I Issues and Problema in the Planning of Education in Developing Countries. cambridge: Harvard University Press 1980.

JENKS, Christopher, et. al. Ineguality. A Reassesment of the Effect of Family and Schooling in America. New York: Harper Colophon Books, 1972.

McGINN, Noei y Ernesto Toro. "Una evaluación de la educación media técnica en El Salvador". Revista Latinoamericana de Estudios Educativos. Vol. X No. 2, 1980.

MEN-SER Evaluación del Rendimiento Externo del Bachillerato Colombiano: Informe final sobre la cohorte de Bachillerato Egresados en el año de 1978. Bogotá, octubre 1982.

PSACHAROPOULOS G. y O. Soumelis. "A quantitatjve analysis of the demand for higher education”. Higler Education Amsterdam: Elsevier, 1979.

SCHIEFELBEIN, E. y J. FARREL. Eight years of their lives. Throygh Schooling to the labor Marlset in Chile Otawa: IDRC, 1982.

VAN de GEER, JOHN. Introduction to Multiuariate Analysis for the Social Sciences. San Francisco: W. M. Freeman and Co. 1971. 